\title{
A Review of Mouse-Tracking Applications in Economic Studies
}

\author{
Geran Tian ${ }^{1,2}$, Weixing $\mathrm{Wu}^{1^{*}}$ \\ ${ }^{1}$ University of International Business and Economics, Beijing 100029, China \\ ${ }^{2}$ Dyson School of Applied Economics and Management, Cornell University, Ithaca, New York, USA \\ tiangeran@126.com,wxwu@uibe.edu.cn
}

\begin{abstract}
Since mouse-tracking paradigm came under the spotlight two decades ago, by providing mouse cursor trajectories, it has been applied by behavioral scientists to a variety of topics to help understand realtime psychological state when people are faced with multiple choices. In this article, we provide a comprehensive, documentation of experimental economics studies with mouse-tracking paradigm. Among these studies, some focus on measuring choice uncertainty including subject uncertainty, temporal uncertainty, and probabilistic uncertainty; the rest are concerned with economic games including bargaining games and social dilemma games. Why and how these works employ mouse-tracking technique in their experiments is elaborated in detail. Finally, limitations of mouse-tracking paradigm are discussed, and research opportunities are proposed. Basic know-hows are appended as a general guide for interested readers.
\end{abstract}

Keywords: Mouse-tracking, Behavioral decision-making, Experimental economics, Cursor trajectory, Game theory.

\section{Introduction}

Computerized experiments are favored by experimental economists because of the high efficiency and accuracy. To facilitate researchers, toolkits were developed such that little or minor programming is needed for specific experiments. To augment the functions of ready-made platforms, auxiliary tools such as eyetracking and mouse-tracking were developed. As one of the earliest supplementary tools for economic experiments, eye-tracking first appeared in the late $19^{\text {th }}$ century. Over decades, its accuracy, ease of operation, cost, and unobtrusiveness have been ameliorated, and its application in marketing and psychology research has been expanded. However, an eye-tracking system costs $\$ 3,000$ to $\$ 30,000$ depending on configuration. As technology advances, mouse-tracking has emerged in the beginning of the $21^{\text {th }}$ century as an economical alternative to eye-tracking.

Mouse-tracking is a computer-based program that records cursor position information of computer users and offers abundant real-time data of decision-making process, and thus it is also referred to as response dynamic. In contrast to eye-tracking with result error of 31 to 59 pixels (Johansen, San Agustin, Skovsgaard, Hansen, \& Tall, 2011), mouse-tracking records cursor trajectories down to exact pixels. While eye-tracking protocol records discrete data points during trails, averages over trials, and yields time-course estimates of processing, mouse-tracking paradigm provides continuous time-course coordinates, making it possible for dynamical systems theory analysis (Magnuson, 2005). In a typical mouse-tracking experiment, participants are asked to complete tasks on computers, including moving mouse cursor and choosing one or more options among multiple alternatives that are represented by clickable buttons.

As the participants proceed, the motion of cursor is recorded. According to the theories that motor functions are driven by cognitive process (Goodale, Pelisson, \& Prablanc, 1986; Spivey \& Dale, 2006), cursor trajectories are considered as a sign of commitment to or contradiction among available choices (Freeman, Dale, \& Farmer, 2011). Thus, mouse-tracking technique has been utilized as a tool for cognitive science, economics, and computer science studies, where researchers can drill deep into computer users' real-time decision-making process. In this review, we aim to summarize an array of studies with cursor dynamics in economics and how it can be further exploited. The interpretation on those articles focuses on (1) what problem they address and (2) how mouse-tracking technique was utilized, regardless of theoretical frameworks. The literature search for this review was concluded in July 2019. 


\section{Techniques that Capture Cursor Information}

Currently, there are a few software packages available on the internet to acquire mouse cursor information Mouseflow ("Mouseflow," n.d.), Mousetrap (Kieslich \& Henninger, 2017), Heat Map Tracker (Callen \& Thompson, 2019), Clicktale ("Clicktale," n.d.), Lucky Orange ("Lucky Orange," n.d.), MouselabWEB (a web version of Mouselab) (Lohse \& Johnson, 1996; Willemsen \& Johnson, n.d.), MouseTracker (Freeman \& Ambady, 2010), etc. Each has its own expertise for example MouselabWEB is dedicated to monitor webpage searching behaviors, while MouseTracker is developed to trace decision-making process on a speciallydesigned computer program. These toolkits also generate outputs in distinct forms. Heat Map Tracker exhibits tracking results by creating heat maps, while Mousetrap produces average cursor trajectories and tracking parameters. Notably, although "tracing" and "tracking" are almost semantically indifferent, in this paper.

We follow the tradition from previous literature that "tracing" refers specifically to Mouselab, and "tracking" represents the technologies that record the entire mouse cursor trajectories. Mouselab and Mouse-tracking differ significantly in theoretical focus, interface design, and data structure. For MouseTracker, the options are often binary and are placed at the top left and right corners. In most cases, the task either asks the participants to indicate their preferences, or requires the participants to respond to certain stimuli. The cursor trajectory is recorded in three dimensions - $\mathrm{x}, \mathrm{y}$, and time. Namely, for each point of time, a pair of (x, y) coordinate is recorded. Mouse-tracking software's that record cursor trajectories such as MouseTracker are considered sensitive detector of response conflict in a situation facing two options. As for Mouselab, information on the computer is initially masked by boxes, and is often arranged in form of m $\times n$ matrix. Experiment participants can reveal information by either one of the three ways depending on Mouselab settings: 1) moving the mouse cursor inside the box, 2) moving the cursor inside the rectangle and leftclicking the box, 3) moving the cursor into the box and holding the left mouse button down.

Instead of the trajectories, the time that cursor enters and exits the rectangle is reported by Mouselab. In other words, Mouselab focuses on decision process with respect to information acquirement; by contrast, MouseTracker monitors how attention flows. Based on definition of what is recorded, Mouselab does not belong to mouse-tracking, but due to its early and extensive use in game theory studies and the fact that it deals with mouse cursor information, Mouselab is also considered in Section 3 of this review. Apart from Mouselab, decision moving-window (DMW) is another alternative (Franco-Watkins \& Johnson, 2011). DMW can be viewed as a combination of eye-tracking and mouse-tracing. DMW is an eye-tracking protocol with occlusion, meaning the information is masked until the subject fixates on the area. This paradigm is tested with risky choice experiments and the results were compared with those of mouse-tracing and basic eyetracking. It is found that DMW led to fewer fixations than the other two methods (Franco-Watkins \& Johnson, 2011). Notably, Bonn experimental System (BoXS) (Seithe, Morina, \& Glockner, 2016) incorporates mousetracking and eye-tracking options for both interactive and non-interactive experiments. Some of the most important technical know-hows are introduced in the appendix.

\section{Research Articles with Mouse Cursor Information}

Behavioral Economics: In behavioral economics, mouse-tracking is usually employed to detect choice uncertainty. Tzafilkou, Protogeros, and Yakinthos (2014) suggest that risk perception leads to hesitation, whose pattern is one of the most common and salient patterns of all mouse behaviors. Variables were identified as indicators of risk perception: pause time (Dijkstra, 2013), movements between choices (Ferreira, Arroyo, Tarrago, \& Blat, 2010), and response time ("Clicktale User Manual," 2010). To decipher how mouse cursor movement is related to uncertainty, R. Bodily et al. (2015) designed a set of experiments based on Metamemory Framework, which suggests that when people are given a question, they have a feeling of knowing. Feeling of knowing is the extent to which the decision maker thinks he/she knows the answer of the question or he/she can find the answer after comprehending the question but before trying to answer it. However, one may encounter the situation that he/she has a strong feeling of knowing, but cannot find a fine answer. 
The mouse cursor appears at the bottom center of the screen at the beginning of the task. According to this model, there are at least three possible outcomes: 1) the subject knows the answer and responds fast; 2) the subject is not aware of the answer but searches for one; 3) the subject does not know the answer and randomly selects one. Three situations represent different levels of choice uncertainty, ranking from low to high. In the experiments, low, moderate, and high uncertainty questions were deployed and the results showed that AUC was higher when people had moderate level of uncertainty than if low or high uncertainty were present. Uncertainty is tightly embedded in intertemporal choice. In a study of (O'Hora, Carey, Kervick, Crowley, \& Dabrowski, 2016), participants were requested to indicate which they prefer, a money reward now or a larger one later. Decision dynamics revealed by cursor trajectories indicated that as reward delayed more, subjective value of reward decreased.

Those who chose "now" responded faster, made less $\mathrm{x}$-flips, and were less likely to create a large MD. On the other hand, those who chose "delay" when rewards were further postponed exhibited higher MD and more xflips, which is in consistent with the findings from (Dshemuchadse, Scherbaum, \& Goschke, 2013). When money reward is replaced by a "vice" preferred by participant, which might be alcohol, food, cigarette, or nothing, the area under the discounting curve (AUDC) (Note that "area under the discounting curve" is abbreviated to "AUC" in some literature, which differs from "area under the curve") demonstrated interesting results. The participants who chose smoking as their vice exhibited the largest discounting factor $\mathrm{k}(\mathrm{k}=1$ AUDC), and those who did not choose a vice discounted their rewards least strongly. Cheng and GonzálezVallejo (2017) deployed mouse-tracking paradigm on intertemporal choice experiment, conducted principle component analyses on data collected, and suggested that decision-making difficulties are composed of two facets - uncertainty and conflict.

An uncertain situation means the options are similar to the subject, and a conflict situation provides tempting and competing alternative. For example smaller and sooner versus larger and later rewards, Cheng and González-Vallejo (2017) also reported that idle time reflects thinking, instead of distraction, and that x-flip indicates choice uncertainty, instead of jittering. Other than the studies mentioned above, Dshemuchadse et al. (2013) confirmed date-delay effect with their mouse-tracking experiment evidence, i.e. stronger temporal discounting when representing delays (e.g. "in 9 days") comparing to calendar dates (e.g. "on the 11th of November") as measured by beta weights. Apart from temporal uncertainty, Koop and Johnson (2011) and Bruhn (2013) on the other hand examined how mouse cursor reflects probabilistic risks. In their works, cursor trajectory captured the nuance when subjects were faced with different probability and probabilistic cues.

Game Theory: Early game theory studies with mouse cursor information are dominated by Mouselab protocol. With Mouselab, payoffs are concealed by black rectangles, and subjects can reveal the payoffs with the mouse. One of the earliest Mouselab applications in game theory appears in the book written by Camerer, Johnson, Rymon, and Sen (1993) in order to keep an account of information search process in a three-round alternating-offer bargaining game (e.g. a $\$ 5$ pie with a discount factor of 0.5 means in the perfect equilibrium the first mover should offer $\$ 1.25$ and keep the rest). The incentive behind was to inspect how information was assembled in brain, aiming at addressing two questions: (1) what information was retrieved; (2) in what order information was processed. If the participants harnessed backward induction to calculate perfect equilibrium, they should have first inspected third-round information, and then looked at second-round, finally first-round.

Camerer et al. (1993) also predicted longest inspection time in the second-round. As is common in most bargaining games, the offers mostly lied between equal split and perfect game equilibrium. Participants need to move the mouse to reveal his/her role and the pie size for each round. Since the information was revealed as long as mouse cursor stayed upon the box, subjects opened and reopened boxes frequently, rather than memorized the information covered by the boxes. Mouselab suggested that subjects did not follow the expected route from the third-round to the first-round and did not look at information long enough for backward induction. Instead, they focused primarily on current round to make offers. Some subjects looked at future round information and were more likely to accept low offers, but they were not more likely to make offers closer to equilibrium. In this study, researchers deduced that subjects did not adopt backward induction from Mouselab evidence. 
Costa-Gomes, Crawford, and Broseta (2001) altered Mouselab settings in a normal-form game experiment such that the subjects could only reveal information by left-clicking and leave the box by right clicking. This approach reduces the noise caused by random look-ups and guarantees each inspection was a conscious action. Theoretically, mouse-tracking can record more information than Mouselab in aforementioned games with the same setting. But the conundrum is how to interpret noisy trajectories lingering around the boxes, and that is probably why economists chose Mouselab for their games. One intriguing phenomena in social dilemma games is that under some circumstances people deviate from their best response to cooperate, especially when time pressure is present. With the help of mouse-tracking, Kieslich and Hilbig (2014) investigated whether defection, as compared to cooperation, entails more cognitive conflict.

MD was shown to be higher for people who decide to defect than to cooperate in three games - prisoner's dilemma game, chicken game, and stag hunt game, all of which embody the features of social dilemma. This study did not recruit time as an indicator for intuition/reflection, and thus avoid the controversy whether a fast response was intuition-driven in decision making. They further conclude that since cooperation is associated with lower degrees of cognitive conflict and thus it is the "default" choice. Opposing this argument, Myrseth and Wollbrant (2015) reasoned that since cognitive conflict can come from resisting impulse both successfully and unsuccessfully, and defection might be the result of unsuccessful self-control. In recent years, there are more studies exploring the cognitive process underlying economic games. Myrseth and Wollbrant (2015) documented a table describing the full scenario (2015, Table 1), and the following Table 1 is an augmentation of it.

We conjecture that, one cannot tell which choice is the "default" without knowing whether self-control wins or impulse wins, but if economists were able to construct two groups of participants that differ in and only in who wins the battle and redo this mouse-tracking experiment, this problem might have a solution. For those with strong self-control (type A subjects), self-control wins over intuition in most, if not all, decision-making trials, and whichever choice type A subjects made with less conflict should be the "default" choice. And vice versa, type B subject choice with more conflict should be "default". That said, how to select type A and B subjects remains questionable. There might be two ways: (1) by personality tests emphasizing self-control; (2) by different stimulus prior to mouse-tracking experiment. However, personality test can be difficult and dangerous - with personality test, it is difficult to make sure that type A and B are not fundamentally different (A \& B may differ in many other aspects as well.

Finally, there are 4 possible outcomes: (1) for group A, MD(defect) $>\mathrm{MD}$ (cooperate), and for group B, $\mathrm{MD}$ (defect) $<\mathrm{MD}$ (cooperate); (2) for group $\mathrm{A}$, $\mathrm{MD}($ defect $)<\mathrm{MD}$ (cooperate), and for group $\mathrm{B}$, $\mathrm{MD}$ (defect) $>\mathrm{MD}$ (cooperate); (3) for both $\mathrm{A}$ and $\mathrm{B}, \mathrm{MD}$ (defect) $>\mathrm{MD}$ (cooperate); for both $\mathrm{A}$ and $\mathrm{B}$, $\mathrm{MD}$ (defect) $<\mathrm{MD}$ (cooperate). For the outcome 3 and 4, one can easily conclude which is the "default" choice; but if outcome 1 or 2 happens with statistical significance, the pre-treatment is playing a role affecting subjects' "default" choice. The table other than the first column is the full scenario Myrseth and Wollbrant (2015) proposed, and Kieslich and Hilbig's Study (2014) is consistent with highlighted cells. The first column is added to show, how to construct subject samples such that we know which row we are referring to in each trial.

Table 1: Experiment Design for Kieslich and Hilbig's (2014) Social Dilemma Game

\begin{tabular}{|c|c|c|c|}
\hline $\begin{array}{lr}\text { Designed } & \text { Self-Control } \\
\text { Through } & \text { Grouped } \\
\text { Subjects } & \\
\end{array}$ & $\begin{array}{l}\text { Assumed } \\
\text { Self-Control } \\
\text { and its Relation to } \\
\text { Conflict }\end{array}$ & $\begin{array}{l}\text { Assumed “Def } \\
\text { Cooperation }\end{array}$ & Defection \\
\hline $\begin{array}{l}\text { Null (subjects without } \\
\text { self-control do not } \\
\text { provide differentiated } \\
\text { conflict results) }\end{array}$ & No-self control & $\begin{array}{lr}\text { No difference } & \text { between } \\
\text { Cooperators } & \text { and } \\
\text { Defectors } & \end{array}$ & $\begin{array}{l}\text { No difference between } \\
\text { Cooperators and } \\
\text { Defectors }\end{array}$ \\
\hline $\begin{array}{l}\text { Subject type A: } \\
\text { Self-control wins }\end{array}$ & $\begin{array}{l}\text { More conflict with } \\
\text { successful self-control }\end{array}$ & $\begin{array}{lrl}\text { More conflict } & \text { for } \\
\text { Defectors } & \text { than } & \text { for } \\
\text { Cooperators } & & \end{array}$ & $\begin{array}{l}\text { More conflict for } \\
\text { Cooperators than for } \\
\text { Defectors }\end{array}$ \\
\hline
\end{tabular}




\begin{tabular}{lllllll}
\hline $\begin{array}{l}\text { Subject type B: } \\
\text { Impulse wins }\end{array}$ & More conflict with & $\begin{array}{l}\text { More conflict for } \\
\text { unsuccessful self-control }\end{array}$ & $\begin{array}{l}\text { More conflict for } \\
\text { Defectors }\end{array}$ & forators than & for & $\begin{array}{l}\text { Defectors than for } \\
\text { Cooperators }\end{array}$
\end{tabular}

Table 2: A Summary of Experimental Studies with Cursor Information in Economics and Cognitive Science

\begin{tabular}{|c|c|c|}
\hline Domain & Topic & Studies \\
\hline \multirow[t]{3}{*}{$\begin{array}{l}\text { Behavioral } \\
\text { economics }\end{array}$} & Subject uncertainty & $\begin{array}{l}\text { R. Bodily, Harris, S., Jenkins, J., Larsen, R., } \\
\text { Sandberg, D., \& Stokes, S., et al. (2015) }\end{array}$ \\
\hline & Temporal uncertainty & $\begin{array}{l}\text { Calluso, Tosoni, Pezzulo, Spadone, and } \\
\text { Committeri (2015); Cheng and González-Vallejo } \\
\text { (2017); Dshemuchadse et al. (2013); Gabaix, } \\
\text { Laibson, Moloche, and Weinberg (2003); O'Hora } \\
\text { et al. (2016) }\end{array}$ \\
\hline & Probabilistic uncertainty & Bruhn (2013);Koop and Johnson (2011) \\
\hline \multirow[t]{2}{*}{ Game theory } & Bargaining game & $\begin{array}{l}\text { Camerer et al. (1993); M. A. Costa-Gomes and } \\
\text { Crawford (2006); M. Costa-Gomes et al. (2001) }\end{array}$ \\
\hline & Social dilemma game & $\begin{array}{l}\text { Kieslich and Hilbig (2014); Myrseth and } \\
\text { Wollbrant (2015) }\end{array}$ \\
\hline
\end{tabular}

\section{Limitations and Future Research}

Mouse-tracking as a newly emerging technique has been playing a non-negligible role in social science research, especially in experimental studies attempting to evaluate people's attention in preferential or perceptual decision-making process. However, in addition to the variability of mouse devices, one of the major limitations of mouse-tracking paradigm is that, mouse behaviors differ significantly among users, and this introduces much noise in data analysis. For example, most people are right-handed, and the mouse are most likely be placed at the right-hand-side of the users by default; some users have a habit of highlighting text with their cursor, and some tend to leave their mouse idle while scanning information. With a being a Wallstreet elite and B being an alcoholic with no self-control, taken to an extreme), and it is dangerous that type B subjects may not undergo the impulse-conscious battle at all. Third, size congruity effect might be triggered by different font size (Henik \& Tzelgov, 1982).

As for the second approach, randomly selected subjects are given distinct stimulus as pre-treatment to provoke group A's deliberation and group B's intuition before mouse-tracking experiments, and one possible pre-treatment would be asking subjects to solve some arithmetic questions, without and with time pressure for group A and B respectively. Therefore, it is necessary to apply aggregate statistics on a pool of users to infer attention (Navalpakkam et al., 2013). Fischer and Hartmann (2014) also proposed several concerns about interpreting mouse-tracking results in number studies. They pointed out specific mental relationships between space and number. First, the SNARC effect (Spatial-Numerical Association of Response Codes) suggests that small and large numbers are associated with left/lower and right/upper areas, respectively (Dehaene, Bossini, \& Giraux, 1993). Second, odd and even numbers are linked with left and right areas, respectively (Nuerk, Iversen, \& Willmes, 2004).

Fourth, the relative location of each digit in a multi-digit numerical influences its meaning through the placevalue system (Nuerk et al., 2004). All these factors make number-related mouse-tracking experiment a tricky case. A possible solution is to randomize the layout of display among trials so that averaged results absorb these effects. Similar to numbers, abstract direction vocabularies (e.g. north, south, west, east) generate a framing effect on the following spatial orders (e.g. up, down, left, right). (Tower-Richardi, Brunyé, Gagnon, Mahoney, \& Taylor, 2012), and this may have an impact on the un-biasedness of some text-oriented mouse- 
tracking studies. At this point, number and text-related effects have not been quantified systematically. Considering a great portion of economic experiments involve numbers and texts, there stands a research opportunity to fully examine to what degree these effects influence the curvature of mouse trajectories, and whether spatial manipulation can mitigate these problems. For economists, there is still plenty of room to expand mouse-tracking in their research.

For example, We discussed in Section 4 how to improve Kieslich and Hilbig's experiment (2014) with a pretreatment to answer the "default" question in social dilemmas. Mouse-tracking is useful in evaluating bounded rationality as well, such as the case in alternating-offer bargaining game (Camerer et al., 1993) where cursor position showed that people in fact did not use backward induction. Other than bounded rationality, there are much more theories and phenomena to be tested with mouse-tracking technology. Nudge theory, for instance, has not been fully examined with mouse-tracking. Other research opportunities lie in examining the neurophysiologic responses associated with mouse moves and clicks, and clarifying whether mouse-tracking data are supplementary to other neuroscience tools, for example skin conductance, functional magnetic resonance imaging (fMRI), electroencephalography (EEG), etc. Hindy et al. (2009) proposed a new combination of mouse-tracking and transcranial magnetic stimulation (TMS), and such a combination has not been fully explored.

\section{Appendix: Measurements and Analyses}

A1: Response Time (RT), Velocity, and Acceleration Component (AC): The definition of RT is selfrevealing. RT measures the time it takes from showing of a certain task page till a decision is made. Rubinstein's work on web-based experiments (2013) suggests that RT can be an indicator of accuracy for objective questions. The strong correlation between relatively short RT and errors is supported by several well-established experiments, including those of Tversky and Kahneman (1983; 1986). Furthermore, novel visualization methods of mouse-tracking data such as decision landscapes (Zgonnikov, Aleni, Piiroinen, O'Hora, \& di Bernardo, 2017) await discovery. Despite the fact that some participants are intellectually unable to give the right answer, some are intentionally choosing random responses to minimize time and effort they devote in the experiments, especially in experiments without performance incentives. One possible solution is to lock each experiment interface for a reasonable period before one can move on to the next question. How long the locking time should be and whether this practice improves devotion deserves further exploration. Note that if task page contains complicated information to read, RT need to be taken good care of and mouse-tracking result will be inevitably harder to deal with (Stillman, Shen, \& Ferguson, 2018). Velocity of cursor trajectory is measured in "pixels per second" or "degree per second".

Researchers are more interested in peak velocity other than average velocity. Conversely, if two choices are situated up and down, there will be a y-flip if a sudden change of choice occurs. To do that, cursor velocities under different treatments are often plotted over the time course, and higher peak velocity is considered a symbol for greater response activation. Derived from velocity and acceleration, AC is an indicator of how many times cursor acceleration changes direction (Dale \& Duran, 2011):

$$
\mathrm{AC}=\left(\sum H\left[-\left(a_{t}-a_{t-1}\right)\left(a_{t-1}-a_{t-2}\right)\right]\right)-1
$$

Where $a_{t}$ is the acceleration of cursor at time $t$. When acceleration changes direction, the Heaviside function gives 1 . However, even in the simplest trajectory, the cursor accelerates and decelerates for at least once, and thus the summation returns 1 . To correct it, 1 is subtracted from AC calculation to standardize this measurement. Usually, when the competition between responses is greater, subjects are more hesitant during the decision-making process and thus lead to greater MD and AUC. However, it is argued that AUC is a better index of overall attraction towards unselected choice incorporating all time intervals, while MD is a better indicator for maximum attraction, limited to shorter time duration.

A2: Measurements for Spatial Attraction - Area under the Curve (AUC) and Maximum Deviation (MD): AUC and MD are calculated based on the ideal response trajectory, which is defined as the line segment connecting the start point and the selected destination. Therefore, ideal response trajectory is the shortest route to give a certain response. AUC is the geometric area enclosed by recorded trajectory and ideal trajectory (Note that the area on the opposite side of the ideal trajectory is considered negative), and MD is 
calculated as the largest perpendicular deviation between the recorded and the ideal trajectory (Freeman \& Ambady, 2010). AUC is also referred to as "curvature" in some literature. An equivalent measure is absolute average deviation (AAD), which is defined as the average deviation between the ideal response trajectory and the actual trajectory.

A3: Measurements for Complexity - X-Flips and Y-Flips: X-flips and/or y-flips are count-based parameters that measure mouse trajectory complexity. "Flip" refers to a sudden change of cursor trajectory from one choice to another, and sometimes it is referred to as "midflight" in literature. As in most cases two choices are allocated with left-right layout, and there is a sudden change of choice along the x-axis, then it is an x-flip. Both AUC and MD are crucial measurements for spatial attraction. In most experimental studies, the correlation between AUC and MD is 0.8 to 0.9 (Stillman et al., 2018), and replacement of AUC by MD for the same data does not critically change the results (Freeman, Ambady, Rule, \& Johnson, 2008). Regarded as a result of competition between potential targets, flip is one of the most important measures for identifying distraction. x-flips are calculated as below (Dale, Roche, Snyder, \& McCall, 2008):

$$
\mathrm{x}-\text { flips }=\sum H\left[-\left(\triangle x_{t}-\triangle x_{t-1}\right)\left(\triangle x_{t-1}-\triangle x_{t-2}\right)\right]
$$

Where $x_{t}$ represents $\mathrm{x}$-axis coordinate at time t. The Heaviside step function will return 1 when there is a change of directionality along the $\mathrm{x}$-axis. Figure 1 is a schematic presentation of how AUC, MD and $\mathrm{x}$-flip are calculated.

Figure 1

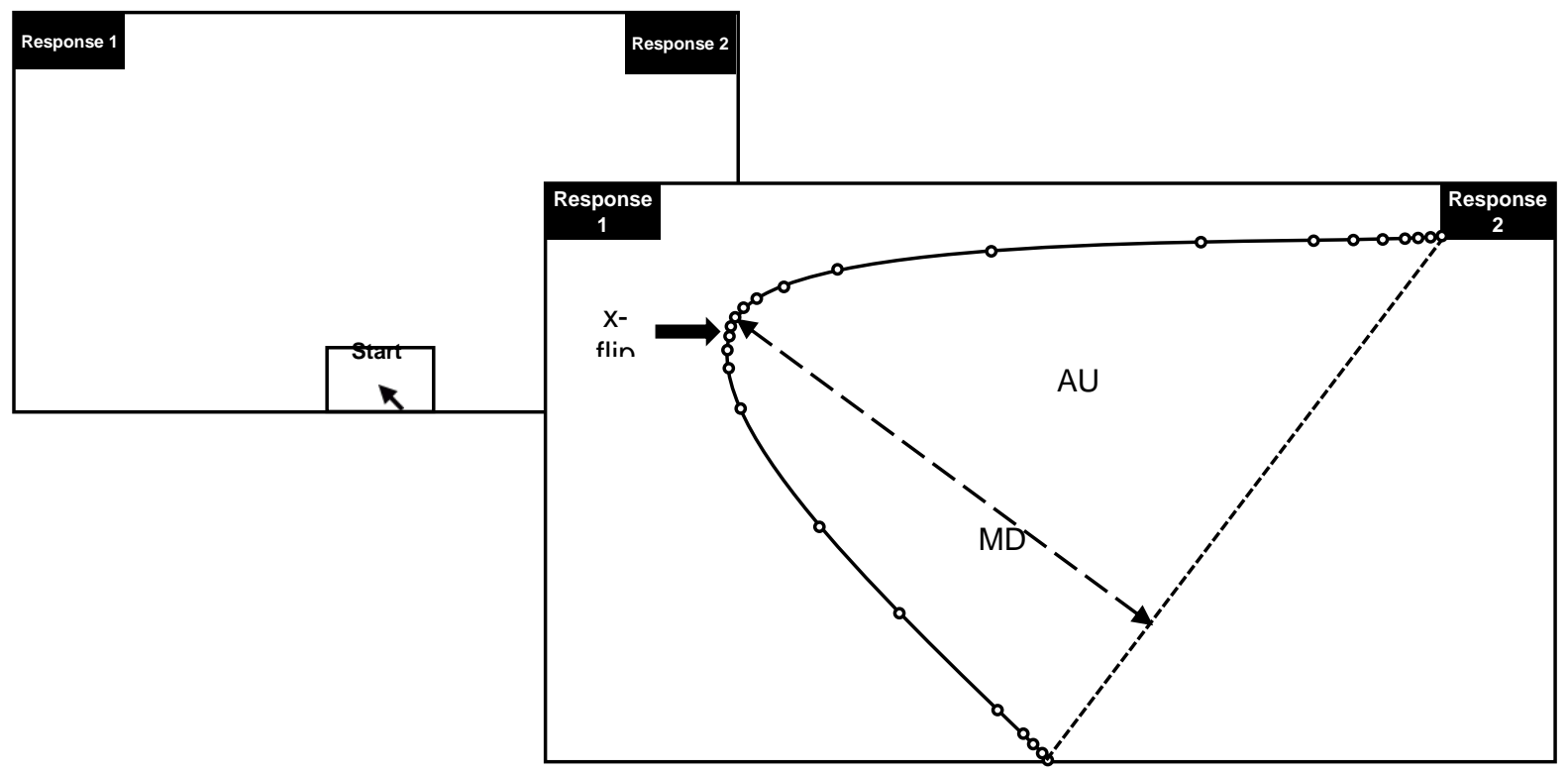

Figure $1 \mathrm{~A}$ schematic presentation on how AUC and MD are defined in a two-way layout. The top-left diagram is initial interface. After clicking "start" button, the task is initiated. The solid curve in the bottom-right diagram represents a possible trajectory, in which case the subject was first attracted to Response 1 and finally chose Response 2, resulting in $1 \mathrm{x}$-flip and corresponding AUC (shaded area) and MD (dotted line).

A4: Distributional Analyses - Bimodality: Bimodality is to detect whether trajectory responses are a result of single- or dual-process cognition. If dual-process is present, some trajectories are likely to exhibit a sudden change, and the distribution of AUC has more than one peak. Freeman and Dale's work (2013) carried out simulations to test several bimodality metrics - Bimodality coefficient (BC), Hartigan's dip (HDS) and the difference in Akaike's information criterion between one-component and two-component Gaussian mixture distribution models, and concluded that the HDS may be the most suitable measure for detecting bimodality for two reasons -HDS is sensitive in distinguishing unimodality and bimodality and it is immune to some disturbing effects. 
Since generally BC and HDS converges, BC's formula is shown below (Cary, 1989):

$$
\mathrm{b}=\frac{g_{1}^{2}+1}{g_{2}+\frac{3(n-1)^{2}}{(n-2)(n-3)}}
$$

Where, $\mathrm{n}$ is the number of observations, $g_{1}$ stands for skewness, and $g_{2}$ represents kurtosis. When b is greater than 5/9, bimodal or multimodal distribution is present. Readers interested in how to analyze dual process cognition with mouse-tracking should read the article of Wel, Sebanz, and Knoblich (2014) for reference.

\section{References}

Bodily, R., Harris, S., Jenkins, J., Larsen, R., Sandberg, D. \& Stokes, S. (2015). A Multi-Experimental Examination of Analyzing Mouse Cursor Trajectories to Gauge Subject Uncertainty. PLoS One, 8(2).

Bruhn, P. (2013). Emergence of spontaneous anticipatory hand movements in a probabilistic environment. Advances in Cognitive Psychology, 9(2).

Callen, M. \& Thompson, M. (2019). Heat Map Tracker. Retrieved from http://heatmaptracker.com

Calluso, C., Tosoni, A., Pezzulo, G., Spadone, S. \& Committeri, G. (2015). Interindividual variability in functional connectivity as long-term correlate of temporal discounting. PLoS One, 10(3).

Camerer, C. F., Johnson, E. J., Rymon, T. \& Sen, S. (1993). Cognition and Framing in Sequential Bargaining for Gains and Losses: Cambridge: MIT Press.

Cary, N. C. (1989). SAS/STAT user's guide. In S. Institute (Ed.).

Cheng, J. \& González-Vallejo, C. (2017). Action Dynamics in Intertemporal Choice Reveal Different Facets of Decision Process. Journal of Behavioral Decision Making, 30, 107-122.

Clicktale. (n.d.). Retrieved from www.clicktale.com

Clicktale User Manual. (2010). In (0.5 ed.).

Costa-Gomes, M., Crawford, V. P. \& Broseta, B. (2001). Cognition and behavior in normal-form games: an experimental study. Econometrica, 69(5), 1193-1235.

Costa-Gomes, M. A. \& Crawford, V. P. (2006). Cognition and behavior in two-person guessing games: An experimental study. American Economic Review, 96, 1737-1768.

Dale, R. \& Duran, N. D. (2011). The cognitive dynamics of negated sentence verification. Cogn Sci, 35(5), 983996.

Dale, R., Roche, J., Snyder, K. \& McCall, R. (2008). Exploring action dynamics as an index of paired-associate learning. PLoS One, 3(3), e1728.

Dehaene, S., Bossini, S. \& Giraux, P. (1993). The Mental Representation of Parity and Number Magnitude. Journal of Experimental Psychology: General, 122(3), 371-396.

Dijkstra, M. (2013). The Diagnosis of Self-Efficacy Using Mouse And Keyboard Input. (Master thesis). Utrecht University,

Dshemuchadse, M., Scherbaum, S. \& Goschke, T. (2013). How decisions emerge: Action dynamics in intertemporal decision making. Journal of Experimental Psychology: General, 142(1), 93-100.

Ferreira, S., Arroyo, E., Tarrago, R. \& Blat, J. (2010). Applying Mouse Tracking to Investigate Patterns of Mouse Movements in Web Forms. Universitat Pompeu Fabra,

Fischer, M. H. \& Hartmann, M. (2014). Pushing forward in embodied cognition: may we mouse the mathematical mind. Frontiers in Psychology, 5(1315).

Franco-Watkins, A. M. \& Johnson, J. G. (2011). Decision moving window: Using interactive eye tracking to examine decision processes. Behavior Research Methods, 43(3), 853-863.

Freeman, J. B. \& Ambady, N. (2010). MouseTracker: software for studying real-time mental processing using a computer mouse-tracking method. Behav Res Methods, 42(1), 226-241.

Freeman, J. B., Ambady, N., Rule, N. O. \& Johnson, K. L. (2008). Will a category cue attract you? Motor output reveals dynamic competition across person construal. J Exp Psychol Gen, 137(4), 673-690.

Freeman, J. B. \& Dale, R. (2013). Assessing bimodality to detect the presence of a dual cognitive process. Behav Res Methods, 45(1), 83-97.

Freeman, J. B., Dale, R. \& Farmer, T. A. (2011). Hand in motion reveals mind in motion. Frontiers in Psychology, 2(59). 
Gabaix, X., Laibson, D. I., Moloche, G. \& Weinberg, S. E. (2003). The Allocation of Attention: Theory and Evidence. SSRN Electronic Journal. doi:doi:10.2139/ssrn.444840

Goodale, M. A., Pelisson, D. \& Prablanc, C. (1986). Large adjustments in visually guided reaching do not depend on vision of the hand or perception of target displacement. Nature, 320, 748-750.

Henik, A. \& Tzelgov, J. (1982). Is three greater than five: The relation between physical and semantic size in comparison tasks. Memory \& Cognition, 10(4), 389-395.

Hindy, N. C., Hamilton, R., Houghtling, A. S., Coslett, H. B. \& Thompson-Schill, S. L. (2009). Computer-mouse tracking reveals TMS disruptions of prefrontal function during semantic retrieval. J Neurophysiol, 102(6), 3405-3413.

Johansen, S. A., San Agustin, J., Skovsgaard, H., Hansen, J. P. \& Tall, M. (2011). Low cost vs. high-end eye tracking for usability testing. Paper presented at the Proceedings of the 2011 annual conference extended abstracts on Human factors in computing systems - CHI EA ' 11.

Kieslich, P. J. \& Henninger, F. (2017). Mousetrap: An integrated, open-source mouse-tracking package. Behav Res Methods, 49(5), 1652-1667.

Kieslich, P. J. \& Hilbig, B. E. (2014). Cognitive conflict in social dilemmas: An analysis of response dynamics. Judgment and Decision Making, 9(6), 510-522.

Koop, G. J. \& Johnson, J. G. (2011). Response Dynamics: How continuous response monitoring can test modern process models. Judgment and Decision Making, 6(8).

Lohse, G. \& Johnson, E. (1996). A comparison of two process tracing methods for choice tasks. Paper presented at the Proceedings of HICSS-29: 29th Hawaii International Conference on System Sciences.

Lucky Orange. (n.d.). Retrieved from https://www.luckyorange.com

Magnuson, J. S. (2005). Moving hand reveals dynamics of thought. Paper presented at the Proceedings of the National Academy of Sciences.

Mouseflow. (n.d.). Retrieved from https://mouseflow.com

Myrseth, K. O. R. \& Wollbrant, C. (2015). Less cognitive conflict does not imply choice of the default option: Commentary on Kieslich and Hilbig (2014). Judgement and Decision Making, 10(3), 277-279.

Navalpakkam, V., Jentzsch, L., Sayres, R., Ravi, S., Ahmed, A. \& Smola, A. (2013). Measurement and modeling of eye-mouse behavior in presence of nonlinear page layouts. Paper presented at the WWW.

Nuerk, H., Iversen, W. \& Willmes, K. (2004). Notational Modulation of the SNARC and the MARC (Linguistic Markedness of Response Codes) Effect. The Quarterly Journal of Experimental Psychology, 57(5), 835863.

O'Hora, D., Carey, R., Kervick, A., Crowley, D. \& Dabrowski, M. (2016). Decisions in Motion: Decision Dynamics during Intertemporal Choice reflect Subjective Evaluation of Delayed Rewards. Sci Rep, 6, 20740.

Rubinstein, A. (2013). Response time and decision making: An experimental study. Judgement and Decision Making, 8(5), 540-551.

Seithe, M., Morina, J. \& Glockner, A. (2016). Bonn eXperimental System (BoXS): An open-source platform for interactive experiments in psychology and economics. Behav Res Methods, 48(4), 1454-1475.

Spivey, M. J. \& Dale, R. (2006). Continuous dynamics in real-time cognition. Current Directions in Psychological Science, 15(5), 207-211.

Stillman, P. E., Shen, X. \& Ferguson, M. J. (2018). How Mouse-tracking Can Advance Social Cognitive Theory. Trends in Cognitive Sciences, 22(6), 531-543.

Tower-Richardi, S. M., Brunyé, T. T., Gagnon, S. A., Mahoney, C. R. \& Taylor, H. A. (2012). Abstract Spatial Concept Priming Dynamically Influences Real-World Actions. Frontiers in Psychology.

Tversky, A. \& Kahneman, D. (1983). Extensional versus intuitive reasoning: The conjunction fallacy in probability judgment. Psychological Review, 90, 293-315.

Tversky, A. \& Kahneman, D. (1986). Rational Choice and the Framing of Decisions. The Journal of Business, 59, 251-278.

Tzafilkou, K., Protogeros, N. \& Yakinthos, C. (2014). Mouse Tracking for Web Marketing: Enhancing User Experience in Web Application Software by Measuring Self-Efficacy and Hesitation Levels. International Journal of Strategic Innovative Marketing.

Wel, R. P., Sebanz, N. \& Knoblich, G. (2014). Do people automatically track others' beliefs? Evidence from a continuous measure. Cognition, 130(1), 128-133.

Willemsen, M. C. \& Johnson, E. J. (n.d.). MouselabWEB. Retrieved from http://www.mouselabweb.org/

Zgonnikov, A., Aleni, A., Piiroinen, P. T., O'Hora, D. \& di Bernardo, M. (2017). Decision landscapes: visualizing mouse-tracking data. Royal Society Open Science, 4(11). 